\title{
Tuberculosis and liver disease: management issues
}

\author{
Ujjwal Sonika, Premashis Kar
}

\section{ABSTRACT}

Department of Medicine, Maulana Azad Medical College,

New Delhi - 110002, India

Correspondence:

Dr. Premashis Kar

Email: premashishkar@gmail.com
Tuberculosis is one of the most common diseases in India and has attained epidemic proportions. Tuberculosis and liver are related in many ways. Liver disease can occur due to hepatic tuberculosis or the treatment with various anti-tubercular drugs may precipitate hepatic injury or patients with chronic liver disease may develop tuberculosis and pose special management problems. Tuberculosis per se can affect liver in three forms. The most common form is the diffuse hepatic involvement, seen along with pulmonary or miliary tuberculosis. The second is granulomatous hepatitis and the third, much rarer form presents as focal/local tuberculoma or abscess. Tubercular disease of liver occurring along with pulmonary involvement as in disseminated tuberculosis is treated with standard regimen for pulmonary tuberculosis. Granulomatous hepatitis and tubercular liver abscess are treated like any other extra-pulmonary tubercular lesions without any extra risk of hepatotoxicity by anti-tubercular drugs. Treatment of tuberculosis in patients who already have a chronic liver disease poses various clinical challenges. There is an increased risk of drug induced hepatitis in these patients and its implications are potentially more serious in these patients as their hepatic reserve is already depleted. However, hepatotoxic anti-tubercular drugs can be safely used in these patients if the number of drugs used is adjusted appropriately. Thus, the main principle is to closely monitor the patient for signs of worsening liver disease and to reduce the number of hepatotoxic drugs in the anti-tubercular regimen according to the severity of underlying liver disease.

KEYWORDS: tuberculosis, chronic liver disease, granulomatous hepatitis

\section{Introduction}

Tuberculosis is one of the most common diseases in India and has attained epidemic proportions. With 3.4 million cases, India carries more than $20 \%$ of the world's TB burden. ${ }^{1}$ However TB is not just a public health problem but also a socioeconomic challenge. The stigma associated with TB in communities has drastic consequences for women and children. TB takes a toll not only on the family but also on the community. An afflicted person infects approximately 12 other people in his lifetime. More than 70\% of TB cases occur in the age group of 15-54 years, which constitutes are primary workforce. Treatment of tuberculosis involves the use of a combination of drugs to prevent drug resistance. These drugs are provided through various Directly Observed Treatment Short-course (DOTS) centres where therapy is given to patients under direct supervision. Although this treatment strategy of using a combination of drugs is highly effective in treating tuberculosis, it may also lead to increased toxicity and side effects. Hepatotoxicity caused by anti-tubercular drugs is one such effect which gets aggravated by the cumulative toxicity of these drug combinations. 


\section{Liver and tuberculosis}

Tuberculosis and the liver can be related in more than one way. There can be direct hepatic involvement by the disease itself, which is common but rarely causes marked impairment of hepatic functions. ${ }^{2}$ Occasionally, local signs and symptoms may be prominent in hepatic tuberculosis, and may constitute the initial or sole presenting feature of the disease. However, even in developing countries, liver tuberculosis accompanied by local symptoms is an uncommon entity. Hepatic tuberculosis presents in three forms. ${ }^{3}$ The most common form is the diffuse hepatic involvement seen along with pulmonary or miliary tuberculosis in 50 to $80 \%$ of patients who succumb to pulmonary tuberculosis. Despite the diffuse involvement of the liver symptoms of liver disease are often absent. The second form is a diffuse hepatic infiltration without recognizable pulmonary involvement, also known as granulomatous hepatitis. The third much rarer form presents as a focal/local tuberculoma or abscess. Tubercle bacilli reach the liver by way of hematogenous dissemination. The portal of entry in the case of miliary tuberculosis is through the hepatic artery whereas in the case of focal liver tuberculosis it is via the portal vein. Irrespective of the mode of entry, the liver responds by granuloma formation. Tuberculous granulomata ${ }^{4}$ are most frequently found in the periportal areas (zone 1 of Rappaport) but may occasionally occur in zone 3 as well. ${ }^{4}$ Both caseating and non-caseating granulomas are seen. In focal tuberculosis, various granulomas may coalesce to form a large tumour like tuberculoma. A tuberculoma which has undergone extensive caseation and liquefactive necrosis forms a tubercular abscess. In a study from western India, a total of 280 consecutive patients with tuberculosis were evaluated prospectively for the etiology and pattern of liver involvement. Of these, 38 patients (15.7\%) had hepatobiliary tuberculosis, whereas 20 patients $(9 \%)$ had other liver diseases. Thirty-eight cases were further classified as follows: (A) hepatic TB (52.6\%):(1) granulomatous hepatitis $26.3 \%$, (2) liver abscesses or pseudotumors - $26.3 \%$ and (3) calcified hepatic granuloma - (0\%); (B) biliary TB (39.4\%): (1) biliary strictures $-5.2 \%$, (2) gall bladder involvement $-2.6 \%$ and (3) biliary obstruction due to lymph node masses - 31.5\%; (C) mixed variety $(7.8 \%)$ : (1) simultaneous granulomatous hepatitis and biliary stricture - $2.6 \%$ and (2) simultaneous lymph node involvement and calcified hepatic granuloma - 5.2\%. All the cases were prescribed standard anti-tuberculosis therapy (ATT) and responded well to treatment. ${ }^{5}$ Cumulative mortality for hepatic tuberculosis ranges between 15 and $42 \%{ }^{2,6}$ The factors associated with adverse prognosis are: age $<20$ years, miliary tuberculosis, concurrent steroid therapy, AIDS, cachexia, associated cirrhosis and liver failure. Even in patients with AIDS and tuberculosis, the cause of death is invariably the former. ${ }^{7}$

Treatment for tuberculosis can lead to hepatic injury by various hepatotoxic anti- tubercular drugs. Rifampicin, pyrazinamide, isoniazid, ethionamide and PAS are all hepatotoxic drugs. ${ }^{8}$ It manifests clinically as symptoms of jaundice, nausea, vomiting and abdominal pain or as raised bilirubin and hepatic transaminases levels. Hepatotoxicity is the most common adverse effect leading to interruption of therapy. ${ }^{9}$ It is associated with a mortality rate of $6-12 \%$ if the drugs are continued after the onset of symptoms. ${ }^{10}$ The risk of hepatotoxicity is increased when the drugs are combined. However, the risk is comparatively lower among patients receiving intermittent, thrice-weekly treatment from DOTS centres. ${ }^{11-12}$

Last but not the least, patients with chronic liver disease can also develop tuberculosis and pose special management problems. Since many of the potent anti-tubercular drugs are hepatotoxic, they may aggravate the underlying disease process. Thus, drug regimens may need to be modified to prevent further hepatic insult. Besides this chronic liver disease is also one of the most important risk factor for the development of ATT induced hepatitis. ${ }^{13}$ Apart from chronic liver disease, it has also been observed that elevated liver enzymes during anti-tubercular therapy occur more commonly in HBsAg carriers than in healthy controls. ${ }^{14}$ Another study has reported that anti tubercular drug induced liver injury is more common in hepatitis $\mathrm{C}$ virus (HCV) infected patients. ${ }^{15}$ Hepatic dysfunction can also alter absorption and distribution of drugs that are metabolized or excreted in the liver such as isoniazid and rifampicin. ${ }^{16}$ Therefore, in the presence of severe liver disease, it is advisable to include fewer hepatotoxic drugs and to extend the period of treatment. ${ }^{17}$ It is also noteworthy that peritoneal tuberculosis is difficult to diagnose in the presence of concomitant liver disease. The ascitic fluid in peritoneal tuberculosis is characterized by increased protein $(>2.5 \mathrm{~g} / \mathrm{L})$, low SAAG $(<1.1 \mathrm{~g} / \mathrm{L})$ and raised LDH ( $>90 \mathrm{U} / \mathrm{L})$ levels. With coexistent chronic liver disease, a lower protein and higher SAAG levels in the ascitic fluid may confound the diagnosis of tubercular ascites, but LDH levels of $>90 \mathrm{U} / \mathrm{L}$ may prove a useful screening tool. ${ }^{18}$ Diagnosis is best confirmed by acitic fluid adenosine deaminase levels or in a few cases by laparoscopy with peritoneal biopsy and $M$. tuberculosis culture. Chronic liver disease also increases the risk of activation of latent tuberculosis as it is an 
immunocompromised state. On the other hand, hepatic involvement by TB can lead to acceleration of underlying liver injury in patients with liver disease.

\section{Management of hepatic tuberculosis}

Tubercular disease of liver occurring along with pulmonary involvement as in disseminated tuberculosis is treated with standard regimen for pulmonary tuberculosis. Granulomatous hepatitis and tubercular liver abscess are treated like any other extra pulmonary tuberculosis lesion. Chemotherapy with standard anti-tuberculosis drugs remains the corner stone of treatment. The standard regimen which consists of four drugs (INH, rifampicin, ethambutol and pyrazinamide) during the initial two months, followed by INH and rifampicin for the next four to six months is used. ${ }^{19-20}$ The above given recommendations have been endorsed by the CDC for the management of tuberculosis in patients with HIV infection. ${ }^{21}$ The hepatic involvement by tuberculosis does not lead to increased risk of hepatotoxicity due to anti-tubercular drugs even with the widespread use of rifampicin and isoniazid in combination. ${ }^{16,22}$ The exact reasons for this are unknown but this may be due to the small number of patients with hepatic tuberculosis rather than a true absence of hepatotoxicity in such cases. However, it suggests that the relative safety of even four drug regimens in hepatic tuberculosis with the overall risk of drug induced liver disease is the same as that associated with treatment of pulmonary tuberculosis. Thus no alterations in the standard drug regime are required to treat hepatic tuberculosis. In addition to chemotherapy anecdotal reports of successful percutaneous drainage of tuberculous liver abscesses have also been noted. ${ }^{23}$

\section{Management of tuberculosis in patients with underlying liver disease}

Treatment of tuberculosis in patients with liver disease poses various clinical problems. First of all there is an increased risk of drug induced hepatitis in these patients. Besides that, implications of drug-induced hepatitis in this group of patients are potentially more serious as their hepatic reserve is already depleted. However, it has been shown that hepatotoxic antitubercular drugs may be safely used in the patients with chronic liver disease including compensated cirrhosis if the number of hepatotoxic drugs used is adjusted appropriately. ${ }^{24}$ Thus the main principle is to reduce the number of hepatotoxic drugs from the treatment regimen and to increase the duration of treatment.
Among the first-line anti-tubercular drugs, pyrazinamide is considered to be the most hepatotoxic and should be avoided in patients with liver disease. ${ }^{25}$ Isoniazid and rifampicin are also hepatotoxic but their combination is more toxic than either drug alone. Among second line drugs PAS is hepatotoxic. Most cases of the anti-tubercular drug related liver injury occurs within 2-3 months of starting the treatment. ${ }^{22,26}$ The drugs which can be safely used in patients with liver disease include aminoglycosides, ethambutol, quinolones and cycloserine. ${ }^{27}$

Monitoring the degree of drug induced hepatic injury is also difficult in this group of patients as fluctuations in the biochemical indicators of liver function related to the preexisting liver disease act as a confounding factor. Thus it becomes difficult to decide whether the derangements in liver function tests are due to the ATT drugs or are a manifestation of the already existing liver disease. However, most patients with preexistent liver disease can tolerate standard tuberculosis treatment regimens with careful monitoring for hepatotoxicity.

\section{Management strategy}

The overall management strategy in this specific group of patients can be divided into two components: (a) monitoring for drug induced liver injury, and (b) modifying the drug therapy.

\section{Monitoring}

Monitoring hepatic function is a very important measure in liver disease patients who are initiated on anti-tubercular therapy. This allows early detection of any hepatic injury and thus permits withdrawal of potentially hepatotoxic drugs before serious life threatening manifestations develop. Patients should be frequently monitored clinically for development of new symptoms such as fatigue, myalgias, nausea, abdominal pain, fever and jaundice. Besides this, regular biochemical investigations should also be performed. Liver function tests should be carried out before initiating ATT, for baseline levels ${ }^{28}$ as it has been seen that patients with abnormal baseline transaminases levels are at increased risk of developing hepatic injury eventually. ${ }^{26}$ These should be repeated twice weekly for the first two weeks followed by weekly monitoring till the end of two months and then monthly investigations till the end of the treatment. ${ }^{29}$

\section{Modified drug therapy}

Anti-tubercular therapy needs to be modified in patients with 
liver disease and fewer hepatotoxic drugs should be included in the regimen. Patients with stable liver disease such as Child's grade A cirrhosis or MELD score $<18$ can be treated on regimes including two potentially hepatotoxic drugs. Patients with Child's grade B cirrhosis or MELD score 18-25 should be treated with only one hepatotoxic drug and patients with advanced liver disease such as Child's grade C cirrhosis or MELD score $>25$ should be treated with regimen containing only non-hepatotoxic drugs. The drug therapy can thus be modified in following ways: ${ }^{30}$ (a) regimes with only two potentially hepatotoxic drug e.g. (1) regimes without pyrazinamide (PZA), (2) regimes without rifampicin (RIF), and (3) regimes without isoniazid (INH); (b) regimes with only one potentially hepatotoxic drug; and (c) regimes with no potentially hepatotoxic drugs.

\section{Regimes without PZA}

Pyrazinamide is considered to be the most hepatotoxic drug and thus this is considered to be the most preferred regime for use in liver disease patients. If PZA cannot be included in the initial phase of treatment then it should consist of at least INH, RIF, and EMB given daily for 2 months; followed by INH and RIF for 7 months in the continuation phase. ${ }^{31}$

\section{Regimes without RIF}

Rifampicin is considered to be the most effective drug against TB and thus should be used whenever possible. An ofloxacinbased regime has been studied in patients with CLD. The regime containing INH+PZA+EMB+OFL for 2 months followed by $\mathrm{INH}+\mathrm{EMB}+\mathrm{OFL}$ for 10 months was found to be less hepatotoxic and better tolerated than rifampicin containing regimes. ${ }^{32}$

\section{Regimes without INH}

Patients can also be treated with RIF, EMB, and PZA for 6 months. Although this regime has two potentially hepatotoxic medications, it has the advantage of retaining a 6-month treatment duration. The data supporting this regime is based on trials evaluating INH resistance. These trials showed high levels of efficacy with 6-month regimes if the initial phase contained four drugs and RIF was used throughout the 6 months. ${ }^{33}$

\section{Regimens with only one potentially hepatotoxic drug}

These regimes should be reserved for patients with severe liver disease. Rifampicin should be retained in these regimes thus avoiding both INH and PZA among the first line drugs. RIF plus EMB, can be given for 12 months, preferably with another agent, such as a fluoroquinolone, for the first 2 months. However, there is no data to support this recommendation. ${ }^{31}$ In another such regime isoniazid, ethambutol and streptomycin can be given for 2 months followed by 10 months of isoniazid and ethambutol thus avoiding rifampicin and pyrazinamide. ${ }^{30}$

\section{Regimens with no potentially hepatotoxic drug}

Patients with severe unstable liver disease should not be given any hepatotoxic drug. The various drugs which can be used in a combination regimen are ethambutol, aminoglycosides, fluoroquinolones, ethionamide, cycloserine and other newer non-hepatotoxic drugs. At least 3 antitubercular drugs should be used in any combination regime. The duration of therapy should be around 18 to 24 months. One such possible regime is a combination of streptomycin, ethambutol and a fluoroquinolone for 18-24 months. ${ }^{30}$ However, there are no clinical trials to prove the efficacy of such combinations.

\section{Treatment of tuberculosis in liver transplant recipients}

Tuberculosis causes substantial morbidity and mortality in liver transplant recipients. The prevalence of active TB infection in transplant recipients is around $1.3 \%$. Among recipients who develop active TB infection, extra-pulmonary involvement is more common (67\%), including multi-organ disease (27\%). The short-term mortality rate is also high among these patients (31\%). Compared with general population, liver transplant recipients have an 18-fold increase in prevalence of active MTB infection and a 4 -fold increase in case-fatality rate. ${ }^{34}$ Orthotopic liver transplant patients also have poor tolerance for conventional antitubercular therapy due to inherent toxicity of these agents and their concomitant bouts of organ rejection. These patients should be given lesser number of hepatotoxic drugs especially those who develop hepatotoxicity during induction. ${ }^{35}$ Isoniazid treatment for latent tuberculosis in this group of patients was found to be associated with reduced TB reactivation and isoniazid-related hepatotoxicity occurred in $6 \%$ of treated patients, with no reported deaths. ${ }^{34}$ Thus for high-risk transplant candidates, isoniazid appears safe and is probably effective at reducing tuberculosis reactivation. 


\section{References}

1. World Health Organization. TB/HIV in the South-East Asia Region Status Report. Regional Meeting of National TB Programme Managers, WHO/SEARO, New Delhi, India. Geneva: WHO. 2009; Nov 2-5:pp. 2-3.

2. Essop AR, Posen JA, Hodkinson JH, Segal I. Tuberculosis hepatitis: a clinical review of 96 cases. QJ Med. 1984;53:465-77.

3. Spiegel CT, Tuazon CU Tuberculosis liver abscess. Tubercle. 1984;65:127-31.

4. Reynolds TB, Campra JL, Peters RL. Hepatic granulomata. In: Zakim D, Boyer TD, editors. Hepatology - A textbook of liver disease. 2nd ed. Philadelphia: WB. Saunders; 1990. p.1098.

5. Amarapurkar DN, Patel ND, Amarapurkar AD. Hepatobiliary tuberculosis in western India. Indian J Pathol Microbiol. 2008;51:175-81.

6. Alvarez SZ, Carpio R. Hepatobiliary tuberculosis. Dig Dis Sci. 1983;28:193-200.

7. Fauci AS, Lane HC. The acquired immunodeficiency syndrome. In: Wilson JD et al, editors. Harrison's principles of internal medicine. 12th ed. New York: McGraw Hill; 1991. p. 1402.

8. Blumberg HM, Burman WJ, Chaisson RE, Daley CL, Etkind SC, Friedman LN, et al American Thoracic Society/Centers for Disease Control and Prevention/Infectious Diseases Society of America: treatment of tuberculosis. Am J Respir Crit Care Med. 2003;167:603-62.

9. Schaberg T, Rebhan K, Lode H. Risk factors for side effects of isoniazid-rifampicin and pyrazinamide in patients hospitalized for pulmonary tuberculosis. Eur Respir J. 1996;9:2026-30.

10. Dash LA, Comstock GW, Flynn PG. Isoniazid preventive therapy: Retrospect and prospect. Am Rev Respir Dis. 1980;121:1039-44.

11. Dhingra VK, Rajpal S, Aggarwal N, Aggarwaln JK, Shadab K, Jain SK. Adverse drug reactions observed during DOTS. J Commun Dis. 2004;36:251-9.

12. Hong Kong Chest Service/British Medical Research Council. First report: Controlled trial of four thrice weekly regimens and a daily regimen all given for 6 months for pulmonary tuberculosis. Lancet. 1981;1:171-4.

13. Rom WN \& Garay SM. Tuberculosis. Philadelphia: Lippincott Williams \& Wilkins; 2004.

14. Lee BH, Koh WJ, Choi MS, Suh GY, Chung MP, Kim H, et al. Inactive hepatitis B surface antigen carrier state and hepatotoxicity during antituberculosis chemotherapy. Chest. 2005;127:1304-11.

15. Ungo JR, Jones D, Ashkin D, Hollender ES, Bernstein D, Albanese $\mathrm{AP}$, et al. Antituberculosis drug-induced hepatotoxicity. The role of hepatitis $\mathrm{C}$ virus and the human immunodeficiency virus. Am J Respir Crit Care Med. 1998;157:1871-6.

16. Kimerling ME, Phillips P, Patterson P, Hall M, Robinson CA, Dunlap NE. Low serum antimycobcterial drug levels in nonHIV infected tuberculosis patients. Chest. 1998;113:1178-83.

17. Sahbazian B, Weis SE. Treatment of Active Tuberculosis: Challenges and Prospects. Clin Chest Med 2005;26:273-82, vi.

18. Shakil AO, Korula J, Kanel GC, Murray NG, Reynolds TB. Diagnostic features of tuberculous peritonitis in the absence and presence of chronic liver disease: a case control Study. Amer J Med. 1996;100:179-85.
19. Plumber ST, Pipalia DH, Vora IM, Bhambhure N, Naik SR. Hepatic granulomas: profile and follow-up of 10 cases responding to antituberculous therapy. J Assoc Physician India. 1987;35:207-9.

20. Maharaj B, Leary WP, Pudifin DJ. A prospective study of hepatic tuberculosis in 41 black patients. Q J Med. 1987;63:517-22.

21. Centres for Disease Control. Diagnosis and management of mycobacterial infection and disease in persons with human immunodeficiency virus infection. Ann. Intern. Med. 1987;106:254-6.

22. Grönhagen-Riska C, Hellstrom PE, Fröseth B. Predisposing factors in hepatitis induced by isoniazid-rifampin treatment of tuberculosis. Am Rev Respir Dis. 1978;118:461-6.

23. Mustard RA, Mackenzie RL, Gray RG. Percutaneous drainage of a liver abscess. Can J Surg. 1986;29:449-50.

24. Park WB, Kim W, Lee KL, Yim JJ, Kim M, Jung YJ, et al. Antituberculosis drug-induced liver injury in chronic hepatitis and cirrhosis. J Infect. 2010;61:323-39.

25. World Health Organisation. Treatment of tuberculosis: Guidelines for National Programme. 3rd edition. Geneva. 2003. p. 27-38.

26. Teleman MD, Chee CB, Earnest A, Wang YT . Hepatotoxicity of tuberculosis chemotherapy under general programme conditions in Singapore. Int J Tuberc Lung Dis. 2002;6:699-705.

27. Garg PK, Tandon RK. Antituberculosis treatment induced hepatotoxicity. In: Sharma SK, Mohan A, editors. Tuberculosis. I st ed. New Delhi: Jaypee; 2001. p. 500-6.

28. American Thoracic Society and Centers for Disease Control and Prevention. Targeted tuberculin testing and treatment of latent tuberculosis infection. Am $J$ Respir Crit Care Med. 2000;161:S221-47.

29. Saukkonen JJ, Cohn DL, Jasmer RM, Schenker S, Jereb JA, Nolan CM, et al. An official ATS statement: hepatotoxicity of antituberculosis therapy. Am J Respir Crit Care Med. 2006;174:935-52.

30. WHO treatment of tuberculosis guidelines. 4th edition. 2010. p. 96-7.

31. American thoracic society, $\mathrm{CDC}$, infectious disease society of America. Treatment of tuberculosis. Morbidity and mortality weekly report: recommendations and reports. 2003;52(RR-11):1-77.

32. Saigal S, Agarwal SR, Nandeesh HP, Sarin SK. Safety of an ofloxacin-based antitubercular regimen for the treatment of tubrculosis in patients with underlying chronic liver disease: a preliminary report. J Gastroenterol Hepatol. 2001;16:1028-32.

33. Mitchison DA, Nunn AJ. Influence of initial drug resistance on the response to short-course chemotherapy of pulmonary tuberculosis. Am Rev Respir Dis. 1986;133:423-30.

34. Holty JE, Gould MK, Meinke L, Keeffe EB, Ruoss SJ. Tuberculosis in liver transplant recipients: a systematic review and meta-analysis of individual patient data. Liver Transplantation. 2009;15:894-906.

35. Meyers BR, Papanicolaou GA, Sheiner P, Emre S, Miller C. Tuberculosis in orthotopic liver transplant patients: increased toxicity of recommended agents; cure of disseminated infection with nonconventional regimens. Transplantation. 2000;69:64-9. 